\title{
Dynamics of a dust crystal with positive and negative dust
}

\author{
Ioannis Kourakis ${ }^{\dagger}$, Padma Kant Shukla ${ }^{\dagger}$ and Gregor Morfill ${ }^{\ddagger}$ \\ $\dagger$ Institut für Theoretische Physik IV, Ruhr-Universität Bochum, D-44780 Bochum, Germany \\ $\ddagger$ Max Planck Inst. für extraterrestrische Physik, Giessenbachstrasse, D-85741 Garching, Germany
}

\begin{abstract}
A dust crystal consisting of charged dust grains of alternating charge sign $(. . . /+/-/+/-$ $/+/ \ldots)$ and mass is considered. Considering the equations of longitudinal motion, a linear dispersion relation is derived from first principles, and then analyzed. Two modes are obtained, including an acoustic mode and an inverse-dispersive optic-like one. The nonlinear aspects of longitudinal dust grain motion are also briefly addressed, via a Boussineq and Korteweg- de Vries description.
\end{abstract}

Introduction. One of the most astonishing novel characteristics of dusty (complex) plasmas is the occurrence of strongly coupled dust configurations, such as the spontaneous formation of crystalline-like periodic arrangements, in the sheath region (above the negative electrode) in gas discharge experiments. Crystal formation and dynamics have been studied in numerous experiments, in which 'dust' particles were essentially created by injecting artificial (e.g. formaldehyde) micro-spheres, which subsequently acquire a fixed (negative, usually) charge via inherent dynamic charging mechanisms. More recent experimental studies have been devoted to studies of alternating charge sign (positive-negative) dust configurations [1].

Formulation of the problem - a model dust bi-crystal. In this study, we consider a one dimensional horizontal chain (assumed infinite, for simplicity) consisting of negative and positive dust grains, located at equidistant sites (lattice constant $r_{0}$ ). Odd (even) sites, i.e. at $x=(2 n+1) r_{0}\left(x=2 n r_{0} ; n \in \mathscr{N}\right)$, are occupied by negative (positive) charge dust grains, of charge $-Q_{1}\left(+Q_{2}\right)$ and mass $M_{1}\left(M_{2}\right.$, respectively); we assume that $M_{1}>M_{2}$, with no loss of generality. Vertical force equilibrium is ensured by (a balance between) gravity and electric/magnetic forces; only longitudinal displacement $\delta x_{n}=x_{n}-n r_{0}$ (where $n \in \mathscr{N}$ ) is permitted in this simplified model.

The electrostatic binary interaction force $F(r)$ exerted on two grains situated at a distance $r$ is derived from a potential function $U(r)$, viz. $F(r)=-\partial U(r) / \partial x$. Considering the (attractive) interaction between first neighbors only, i.e. $r_{n, n+1}=x_{n+1}-x_{n}=r_{0}+$ $\delta x_{n+1}-\delta x_{n}$, we may Taylor expand $U(r)$ around $r_{0}$, to account for grain displacements. We formally have: $F(r) \approx-U^{\prime \prime}\left(r_{0}\right)\left(r-r_{0}\right)-\frac{1}{2} U^{\prime \prime \prime}\left(r_{0}\right)\left(r-r_{0}\right)^{2}-\frac{1}{6} U^{\prime \prime \prime \prime}\left(r_{0}\right)\left(r-r_{0}\right)^{3}$ (the prime denotes differentiation). In the following, we shall set: $U^{\prime \prime}\left(r_{0}\right)=G, U^{\prime \prime \prime}\left(r_{0}\right) / 2=$ $G A$ and $U^{\prime \prime \prime \prime}\left(r_{0}\right) / 6=G B$. The description of our dust crystal dynamics is thus effectively reduced to a problem of longitudinal atom motion in a diatomic chain, characterized by an anharmonic coupling 'spring' potential. Our dust bi-layer may therefore be analyzed by making use of standard analytical tools from solid state physics [2, 3]. 
For the sake of clarity, one may consider a Debye-type interaction potential (energy) $U_{D}(r)=Q_{1} Q_{2} e^{-r / \lambda_{D}} / r \equiv\left(Q_{1} Q_{2} / \lambda_{D}\right) e^{-\kappa} / \kappa$, for which: $U_{D}^{\prime}\left(r_{0}\right)=$ $-\left(Q^{2} / \lambda_{D}^{2}\right) e^{-\kappa}(1+\kappa) / \kappa^{2}, U_{D}^{\prime \prime}\left(r_{0}\right)=+\left(2 Q^{2} / \lambda_{D}^{3}\right) e^{-\kappa}\left(1+\kappa+\kappa^{2} / 2\right) / \kappa^{3}, U_{D}^{\prime \prime \prime}\left(r_{0}\right)=$ $-\left(6 Q^{2} / \lambda_{D}^{4}\right) e^{-\kappa}\left(1+\kappa+\kappa^{2} / 2+\kappa^{3} / 6\right) / \kappa^{4}$, and $U_{D}^{\prime \prime \prime \prime}\left(r_{0}\right)=+\left(24 Q^{2} / \lambda_{D}^{5}\right) e^{-\kappa}(1+\kappa+$ $\left.\kappa^{2} / 2+\kappa^{3} / 6+\kappa^{4} / 24\right) / \kappa^{5}$, where the lattice parameter is $\kappa=r_{0} / \lambda_{D}$.

Equations of motion. Denoting the odd (even) grain displacement, within the $n-$ th pair, by $\delta z_{2 n+1}=z_{n}\left(\delta z_{2 n}=w_{n}\right)$, the resulting equations of motion read:

$$
\begin{aligned}
M_{1} \frac{d^{2} z_{n}}{d t^{2}}= & G\left(w_{n}-2 z_{n}+w_{n-1}\right)+G A\left[\left(w_{n}-z_{n}\right)^{2}-\left(z_{n}-w_{n-1}\right)^{2}\right] \\
& +G B\left[\left(w_{n}-z_{n}\right)^{3}-\left(z_{n}-w_{n-1}\right)^{3}\right] \\
M_{2} \frac{d^{2} w_{n}}{d t^{2}}= & G\left(z_{n+1}-2 w_{n}+z_{n}\right)+G A\left[\left(z_{n+1}-w_{n}\right)^{2}-\left(w_{n}-z_{n}\right)^{2}\right] \\
+ & G B\left[\left(z_{n+1}-w_{n}\right)^{3}-\left(w_{n}-z_{n}\right)^{3}\right] .
\end{aligned}
$$

Linear vibrations. Assuming a plane wave (phonon) solution in the form: $z=Z \exp i\left[(2 n+1) k r_{0}-\omega t\right]+c . c$. (for the heavy negative grains) and $w=$ $W \exp i\left(2 n k r_{0}-\omega t\right)+$ c.c (for the lighter positive grains), one finds that the frequency $\omega$ is related to the wavenumber $k$ via the dispersion relation

$$
\omega_{ \pm}^{2}=\frac{G}{\mu}\left(1 \pm \sqrt{1-\frac{4 \mu^{2}}{M_{1} M_{2}} \sin ^{2} k r_{0}}\right),
$$

where we have defined the reduced mass $\mu=M_{1} M_{2} /\left(M_{1}+M_{2}\right)$. This relation defines

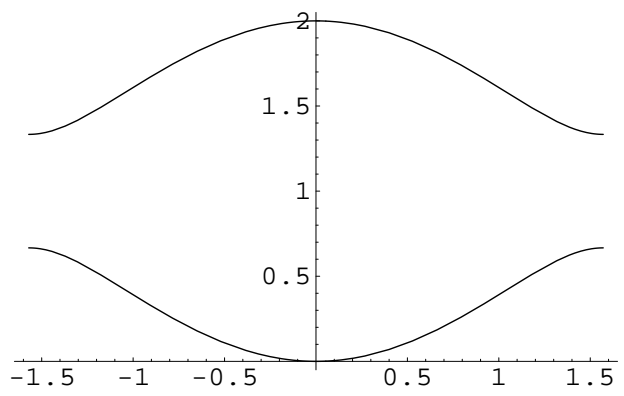

FIGURE 1. The dust bi-layer dispersion relation $\omega_{ \pm}$(normalized by $G / \mu$ ) is depicted vs. the reduced wavenumber $k r_{0}$, for $M 1=2 M_{2}=1$ and $G=1$ (indicative arbitrary values).

a two-fold dispersion curve. The lower branch $\omega_{-}$defines an acoustic mode; at low $k$, it satisfies: $\omega_{-} \approx\left(\frac{2 G}{M_{1}+M_{2}}\right)^{1 / 2} k r_{0} \equiv c_{0} k$, and thus both the group velocity $v_{g r,-}=$ $\omega_{-}^{\prime}(k)$ and the phase velocity $v_{p h,-}=\omega_{-} / k$ tend to the (constant) 'sound velocity' $c_{0}$ for low $k$. The upper branch $\omega_{+}$defines an optic mode; at low $k$, it satisfies: $\omega_{-} \approx$ $\left(\frac{2 G}{M_{1}+M_{2}}\right)^{1 / 2}=$ constant, and thus $v_{g r,+}=\omega_{+}^{\prime}(k)=0$ and $v_{p h,+} \rightarrow \infty$ for long wavelengths $\lambda=2 \pi / k$. The frequency band scanned by the two modes are $\omega_{-} \in\left[0, \sqrt{2 G / M_{1}}\right]$ and 
$\omega_{+} \in\left[\sqrt{2 G / M_{2}}, \sqrt{2 G / \mu}\right]$. We note the appearance of a forbidden frequency range between $\omega_{-}\left(k= \pm \pi / 2 r_{0}\right)=\omega_{-, \text {max }}=\sqrt{2 G / M_{1}}$ and $\omega_{+}\left(k= \pm \pi / 2 r_{0}\right)=\omega_{+, \text {min }}=$ $\sqrt{2 G / M_{2}}$. Furthermore, we point out that the optic mode $\omega_{+}$is characterized by an inverse dispersion, thus $v_{g r,+}=\omega_{+}^{\prime}(k) \leq 0$ everywhere in the first Brillouin zone (1BZ) $\left[0, \pi / 2 r_{0}\right]$. The dispersion curve is depicted in Fig. 1 .

The amplitude eigenmodes - i.e. the solutions of the linearized system of Eqs. (1), for $Z$ and $W-$ satisfy: $W / Z=\left(2 G-M_{2} \omega_{ \pm}^{2}\right) /\left(2 G \cos k r_{0}\right)$. Therefore, in-phase (outof-phase) motion is prescribed for long wavelength acoustic (optic) vibrations, since $W / Z \rightarrow 1\left(W / Z \rightarrow-M_{1} / M_{2}\right.$, respectively) for $k \rightarrow 0$.

Continuum approximation. Assuming a long excitation extension $L \gg r_{0}$, one may substitute the discrete space variables $z_{n}(t)$ and $w_{n}(t)$ with continuous ones, say $z(x, t)$ and $w(x, t)$, by Taylor expanding, i.e. $z_{n \pm 1} \approx z \pm 2 r_{0} z_{x}+2 r_{0}^{2} z_{x x} \pm \frac{4}{3} r_{0}^{3} z_{x x x}+\frac{2}{3} r_{0}^{4} z_{x x x x}+$ $\mathscr{O}\left[\left(2 r_{0} / L\right)^{5}\right]$ (and the analogous expression for $w_{n} \rightarrow w$ ), where the subscript denotes differentiation, e.g. $z_{x}=\partial z / \partial x$ and so forth. Inserting into the discrete equations of motion (1), one thus obtains two coupled partial derivative equations (PDEs). For analytical manipulation purposes, the second one may be neglected by employing the 'Büttner ansatz' [3]: $w \approx \sigma\left[z+b_{1} r_{0} z_{x}+\frac{b_{2}}{2} r_{0}^{2} z_{x x}+\frac{b_{3}}{6} r_{0}^{3} z_{x x x}+\frac{b_{4}}{24} r_{0}^{4} z_{x x x x}+b_{0} f(z)\right]+\mathscr{O}\left(\varepsilon^{5}\right)$, where $\sigma$ is set equal to $1\left(-M_{1} / M_{2}\right)$ for the acoustic (optic) mode, and the parameters $b_{j}$ and the function $f(z)$ are appropriately adjusted for compatibility. One thus remains with one PDE, in terms of $z(x, t)$, while $w(x, t)$ is defined accordingly.

In the following, we shall present some recent results regarding the acoustic mode. The remaining results will be exposed in a detailed article, in preparation.

Nonlinear analysis: the acoustic mode. The compatibility among the eqs. of motion is ensured by choosing [3]: $\sigma=b_{1}=1, b_{2}=2 \mu / M_{2}, b_{3}=6 \mu\left(2 M_{1}-M_{2}\right) /\left(3 M_{1} M_{2}\right)$, $b_{4}=24 \mu\left[1 /\left(3 M_{2}\right)-b_{2}^{2} /\left(4 M_{1}\right)\right]$, and $b_{0}=0$ (for first-neighbor only interactions); see that an ordinary Taylor expansion (viz. $b_{j}=1$ ) is recovered in the limit $M_{1}=M_{2}$.

The system of Eqs. (1) now yield to the nonlinear PDE

$$
z_{t t}-c_{0}^{2} z_{x x}=p_{0} z_{x} z_{x x}+q_{0} z_{x}^{2} z_{x x}+h_{0} z_{x x x x},
$$

or (in an equivalent manner) the Generalized BoUSSINESQ equation

$$
u_{t t}-c_{0}^{2} u_{x x}=p\left(u^{2}\right)_{x x}+q\left(u^{3}\right)_{x x}+h_{0} u_{x x x x},
$$

where we set: $u=z_{x}, p=p_{0} / 2=G A b_{2} / M_{1}, q=q_{0} / 3=G B b_{2} / M_{1}$, and $h_{0}=\frac{2 G}{M_{1}} r_{0}^{4}\left(\frac{b_{4}}{24}-\right.$ $\frac{b_{3}}{6}+\frac{b_{2}}{2}-\frac{1}{3}$ ) (the sound velocity $c_{0}$ was defined above). The ordinary (modified, respectively) BOUSSINESQ equation is recovered from Eq. (4), upon setting $q=0(p=0)$, or $B=0(A=0)$, i.e. by neglecting quartic (cubic) interaction potential contributions.

The GBq Eq. (4) yields two distinct pulse soliton solutions (whose exact form is omitted here, for brevity); these lead (since $u=z_{x}$ ) to the kink (shock-like) soliton:

$$
z(x, t)= \pm 2\left(\frac{6 h_{0}}{q_{0}}\right)^{1 / 2} \arctan \left[\frac{1}{P_{1}} \tanh \left(\frac{x-v t}{L_{1}}+x_{0}\right)\right],
$$

where $P_{1}=\left\{\left[\sqrt{p_{0}^{2}+6\left(v^{2}-c_{0}^{2}\right) q_{0}} \pm p_{0}\right] /\left[\sqrt{p_{0}^{2}+6\left(v^{2}-c_{0}^{2}\right) q_{0}} \mp p_{0}\right]\right\}^{1 / 2} ; x_{0}$ and $v$ are real constants, which determine the soliton center and (supersonic, since $v>c_{0}$ ) velocity, 
respectively; the soliton width is expressed by $L_{1}=2 \sqrt{h_{0} /\left(v^{2}-c_{0}^{2}\right)}$. Recall that $L_{1} \gg r_{0}$ in order for the continuum theory to be valid. The two solutions above correspond to a rarefactive and a compressive localized excitation, propagating in the dust bi-layer.

$K d V$ acoustic soliton theory. By assuming near-sonic propagation, i.e. $v \approx c_{0}$, and a very slow time variation (viz. $u_{\tau \tau} \ll u_{\tau}, u_{\xi}$ ), one obtains from the GBq Eq. (4) the canonical form of the Generalized KORTEWEG - DE VRIES (GKdV) equation

$$
u_{\tau}+6 u u_{\xi}+6 u^{2} u_{\xi}+u_{\xi \xi \xi}=0
$$

[3], where we have defined: $\xi=p_{0}\left(x-c_{0} t\right) / \sqrt{6 h_{0} q_{0}}, \tau=p_{0}^{3} t /\left[2 c_{0}\left(6 q_{0}\right)^{3 / 2} h_{0}^{1 / 2}\right]$, and $u=z \xi \sqrt{q_{0} /\left(6 h_{0}\right)}$. The GKdV Eq. (6) yields two distinct exact soliton solutions, which may be inverted to $u$; one thus obtains two different kink solitons in the form:

$$
z(x, t)= \pm 2\left(\frac{6 h_{0}}{q_{0}}\right)^{1 / 2} \arctan \left[\frac{1}{P_{2}} \tanh \left(\frac{x-v t}{L_{2}}+x_{2}\right)\right],
$$

where $P_{2}=\left\{\left[\sqrt{p_{0}^{2}+12 c_{0}\left(v-c_{0}\right) q_{0}} \pm p_{0}\right] /\left[\sqrt{p_{0}^{2}+12 c_{0}\left(v-c_{0}\right) q_{0}} \mp p_{0}\right]\right\}^{1 / 2} ; x_{2}$ and $v$ are real constants; the soliton width is expressed by $L_{2}=2 \sqrt{h_{0} /\left[2 c_{0}\left(v-c_{0}\right)\right.}$. Again,

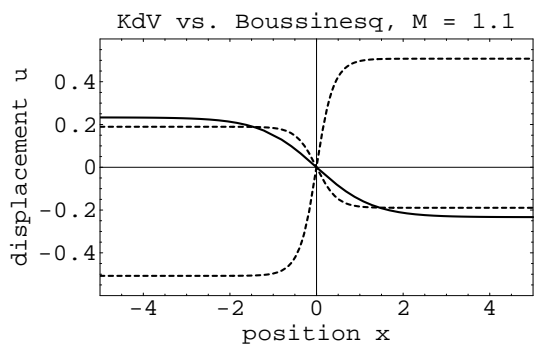

FIGURE 2. Antikink KdV excitation (solid curve) vs. the (two) solutions obtained from the Bq Equation (dashed curves); Debye interactions with $\kappa=1.1$, Mach number $M=v / c_{0}=1.25$.

$L_{2} \gg r_{0}$ is assumed. These two solutions correspond to a rarefactive and compressive localized excitation. Notice that Eq. (7) are recovered from Eq. (5), by setting $v+c_{0} \approx$ $2 c_{0}$. We conclude that the $\mathrm{KdV}$ (and associated) equation related theory adds no extra information to that obtained via the (less approximate) Boussinesq theory.

Similar results have been obtained for one-dimensional dust monolayers [4]. These theoretical considerations will hopefully be confirmed by appropriate experiments.

Acknowledgments. This work was supported by the SFB591 German Programme.

\section{REFERENCES}

1. R.L. Merlino, private communication.

2. C. Kittel, Introduction to Solid State Physics (John Wiley and Sons, New York, 1996).

3. St. Pnevmatikos, Dynamique des Solitons dans les réseaux diatomiques non-linéaires, Thèse d'Etat (Université de Bourgogne, Dijon, 1984).

4. I. Kourakis and P. K. Shukla, Eur. Phys. J. D 29, 247 (2004). 